\title{
HEMATOMA EXTRADURAL TRAUMÁTICO NA INFÂNCIA E TOMOGRAFIA COMPUTADORIZADA PRECOCE NORMAL
}

\author{
RELATO DE DOIS CASOS
}

\author{
ORILDO CIQUINI JR.* - ALBERTO L. MOURA DOS SANTOS ** \\ LUIZ A. MANREZA *** - J. PINDARO PEREIRA PLESE **** \\ RAUL MARINO JR.******
}

\begin{abstract}
RESUMO - São recatados 2 casos de crianças que sofreram traumatismo cranioencefálico (TCE) e cuja tomografia computadorizada craniana (TC), realizada até a terceira hora póstrauma, foi normal. Respectivamente cerca de 20 e 60 horas após a internação, as crianças apresentaram alteração do nível de consciência e nova TC revelou presença de hematoma extradural. Os autores alertam para o fato de que crianças com TCE que apresentam hematomas subgaleais extensos, associados ou não a fraturas da calota craniana, mesmo com* TC normal realizada precocemente, devem permanecer em rigorosa observação pela possibilidade de virem a desenvolver tardiamente hematomas extradurais.
\end{abstract}

PALAVRAS-CHAVE: hematoma extradural, traumatismo cranioencefálico, tomografia computadorizada craniana.

extradural traumatic hematoma in childhood and normal early computed tomographic scan: report of two cases.

SUMMARY - The cases of two children with traumatic extradural hematoma with an early normal CT scan are reported. Consciousness impairment occurred in them respectively 20 and 60 hours after admission, and CT at this occasion evidenced extradural hematoma. Serial neurological examinations are recommended for children "with large traumatic cephalohematoma associated or not to skull fracture and in whom an initial early CT was normal, since they can lately develop extradural hematoma.

KEY WORDS: extradural hematoma, head injury, computed cranial tomographic scan.

Os hematomas extradurais (HED) que ocorrem na infância como complicações de traumatismos cranioencefálicos (TCE) são potencialmente letais, embora infrequentes 3,5,8,9. Após o advento da tomografia computadorizada encefálica (TC), o diagnóstico precoce pode ser firmado, assim como instituído adequado tratamento neurocirúrgico, diminuindo de maneira significativa os índices de mortalidade e morbidade M,9. Smith e Millerio, em publicação recente, chamam a atenção para o fato de que pacientes podem apresentar TC normal logo após o TCE e, tardiamente, desenvolverem HED. Isto é particularmente importante nos traumatismos que acarretam coleções sanguíneas subgaleais associadas a fraturas da calota craniana 3,7 .

Relatamos dois casos em que esta evolução clínica foi observada.

Estudo realizado na Disciplina de Neurocirurgia, Departamento de Neurologia, Faculdade de Medicina da Universidade de São Paulo (FMUSP): * Neurocirurgião; ** Residente de Neurocirurgia; **** Professor Assistente Doutor; $* * * * *$ Professor Associado; ******* Professor Titular.

Dr. J. Píndaro Pereira Plese - Divisão de Neurologia, Hospital das Clínicas, FMUSP - Caixa Postal 8091 - 01065 São Paulo SP - Brasil. 


\section{OBSERVAÇÕES}

Caso 1 - PFV, paciente do sexo masculino, com 10 anos de idade, vítima de quedia de altura superior a 2 metros. O tempo decorrido entre o acidente e a internação foi de cerca de 2 horas. Havia referência de perda momentânea da consciência. O exiame clínico-neurológico de admissão evidenciava escore 15 na escala de coma de Glasgow (GCS), hematoma subgaleal frontotemporal esquerdo (E) e contusão do braço E. A criança estava hemodinamicamente estável. Referia cefaléia pancraniana e apresentou episódios de vômito durante sua admissão. O exame radiológico simples de crânio não evidenciou sinais de fratura dos ossos da calota craniana. A TC com infusão de contraste não evidenciou lesões intracranianas extra ou intra axiais (Fig. IA). Foi instituída terapêutica sintomática e o paciente foi mantido em rigorosa observação clínica. Cerca de 20 horas após a admissão foi observado rebaixamento do nível de consciência com escore 14 na GSC, não sendo detectados sinais neurológicos focais. Nova TC revelou presença de HED frontal E (Fig. 1B), com efeito de massa intracraniana. Foi instituído tratamento neurocirúrgico através de craniotomia: drenagem de HED e coagulação de ramos da artéria meníngea média, origem do sangramento. Não se detectou presença de fraturas da calota craniana durante o ato cirúrgico. A criança recebeu alta no quinto dia pós cirurgia com exame neurológico normal.

Caso 2 - MW, paciente do sexo masculino, com 3 anos e 5 meses de idade, vítima de queda de escada rolante. O tempo decorrido entre o acidente e a internação foi de cerca de 1 hora. Não havia referência de perda de consciência após o traumatismo. O exame clíniconeurológico de admissão revelava criança chorosa, com cefaléia e vômitos, com escore 15 no GSC, hematoma subgaleal frontal E e escoriações faciais. A TC com infusão de contraste foi normal (Fig. 2A). Cerca de 60 horas após o TCE foi notado rebaixamento do nível de consciência sem sinais neurológicos focais e com GSC de 13. Nova TC revelou presença de H.ED frontal E com sinais de herniação intracraniana (Fig. 2B). Foi instituído tratamento neurocirúrgico através de craniotomia: drenagem do HED e coagulação de ramos de artéria meníngea média, origem do sangramento. Não foram detectadas fraturas dos ossos da calota craniana durante $o$ ato cirúrgico. O paciente recebeu alta no quinto dia após cirurgia com exame neurológico normal.
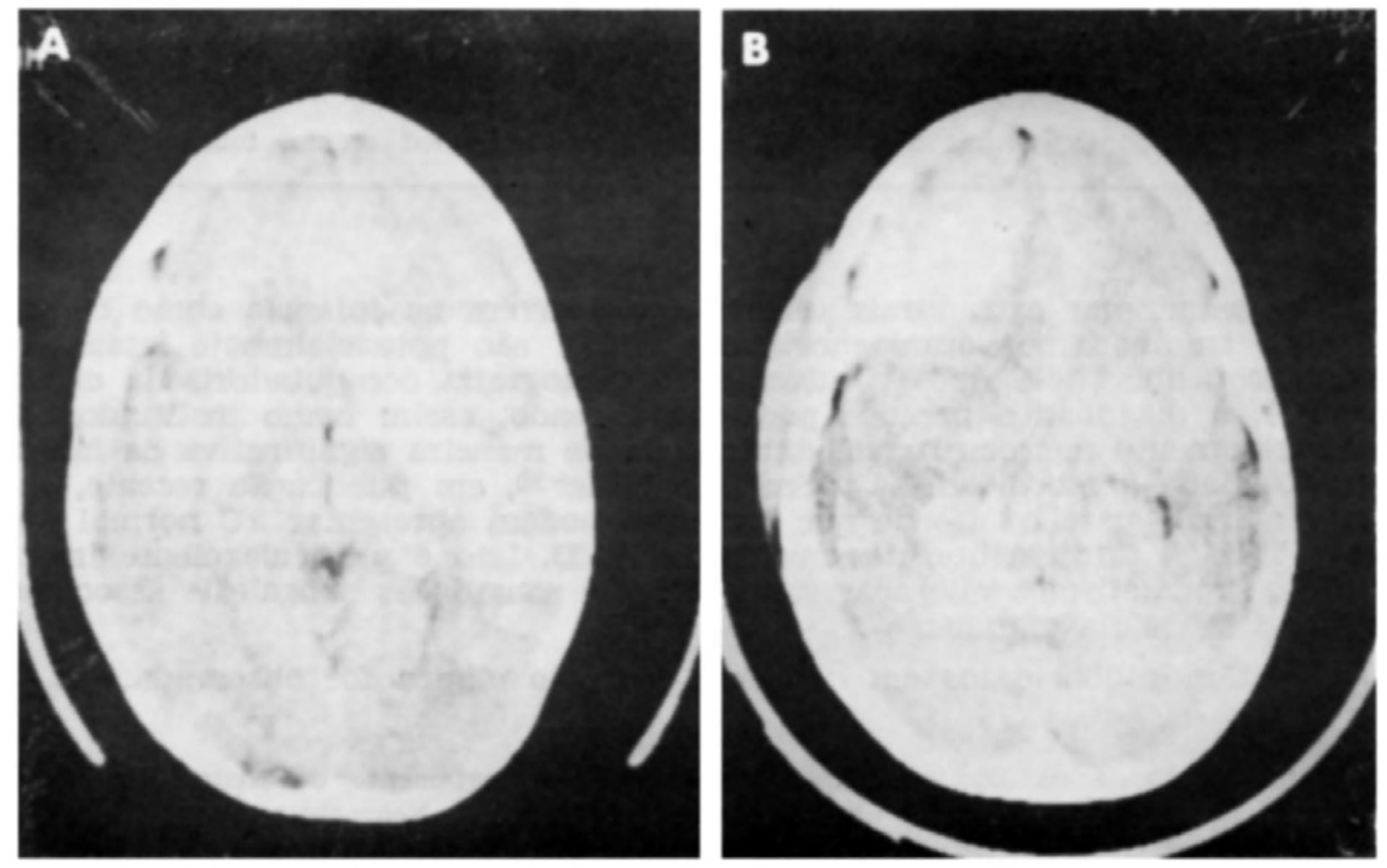

Fig. 1. Caso 1. (A), TC realizada 3 horas apos o TCE: aspecto normal. (B), TC realizada 21 horas apos o TCE: HED frontal esquerdo. 

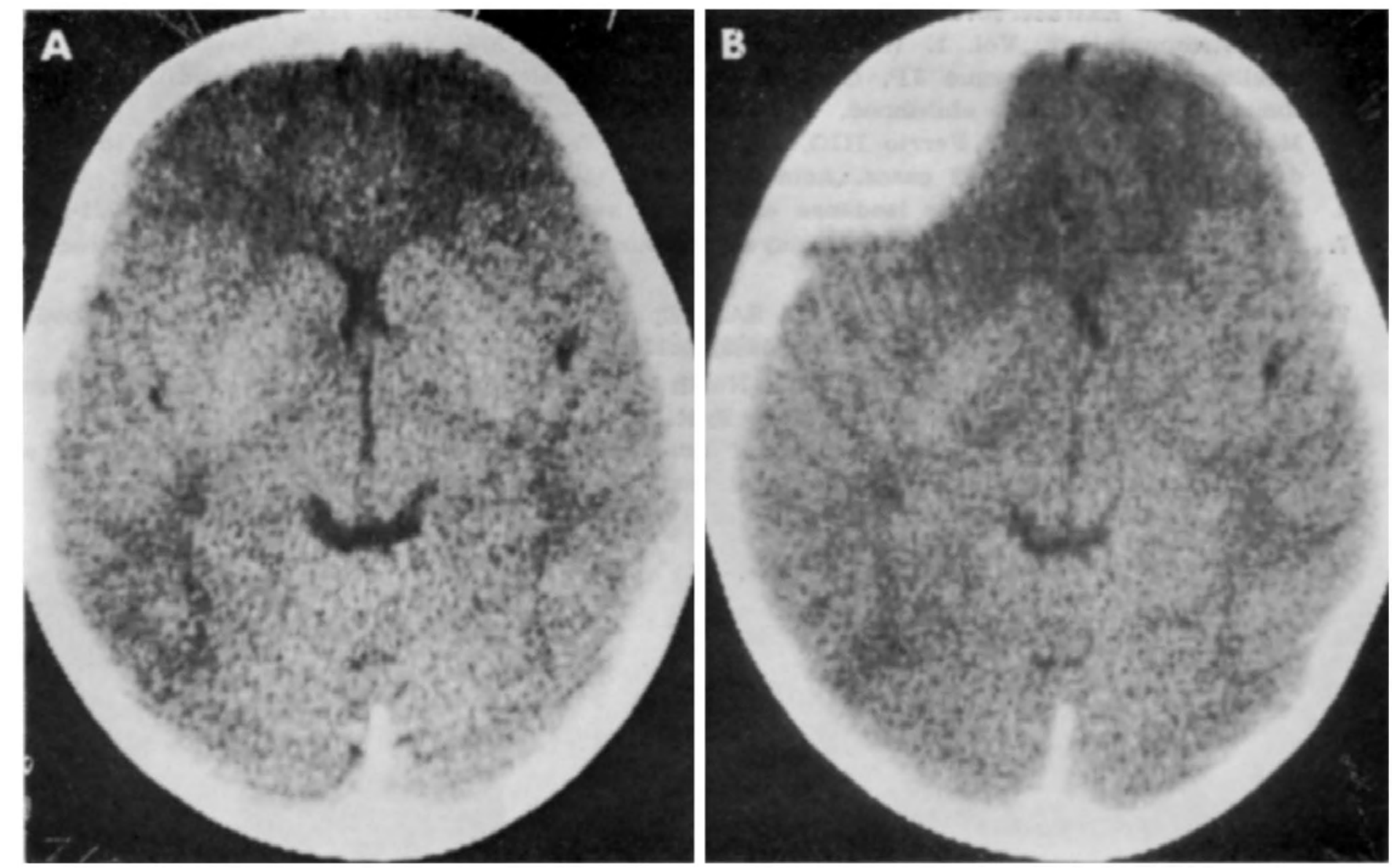

Fig. 2. Caso 2. (A), TC realizada 2 horas após o TCE: aspecto normal. (B), TC realizada 60 horas após o TCE: HED frontal esquerdo.

\section{COMENTÁRIOS}

$O$ pronto atendimento de crianças que sofreram TCE em muito contribuiu para a diminuição do índice de mortalidade e morbidade dessa afecção 3,9 ,io. $O$ diagnóstico precoce do HED por TC permite tratamento eficaz desta complicação do TCE melhorando significativamente o prognóstico destes pacientes, o qual é diretamente relacionado com 0 quadro neurológico no momento da cirurgia i $i{ }^{4} \imath^{5}$.

Cs HED agudos se manifestam clinicamente em período de tempo que varia de alguns minutos a horas quando a origem da hemorragia é arterial ${ }^{2}{ }^{4}>8,9$. Nos casos em que o sangramento é consequente da rotura de veias, o tempo pode ser maior ${ }^{3}$. Traumatismos que acarretam hipotensão arterial sistêmica ou hipertensão intracraniana alteram de maneira significativa a evolução clínica do HED 2.

$O$ intuito deste relato é alertar para o fato de que crianças com TCE, oligossintomáticas, com coleccões sanguíneas subgaleais associadas ou não a fraturas de calota craniana, com CT precoce normal, devem permanecer em observação em serviço especializado, em virtude da possibilidade de desenvolverem HED ou contusões cerebrais tardias 6,7, io. Esta observação deve perdurar por cerca de 24 horas pelo menos em pacientes assintomáticos, ou até que desapareçam os sintomas quando presentes. Frente a qualquer piora do quadro neurológico, mesmo que mínimo, nova TC deve ser indicada para afastar de maneira segura, a ocorrência de HED como complicação do TCE, que pode ser letal ou acarretar graves sequelas se não tratada em tempo hábil.

\section{REFERfiNCIAS}

1. Bricolo AP, Pasut LM. Extradural hematoma: toward zero mortality. Neurosurgery 1984 $14: 8-12$.

2. Bucci MN, Philliphs TW, McGillicuddy JE. Delayed epidural hemorrhage in hypotensive multiple trauma patients. Neurosurgery 1986, 19:65-68. 
3. Choux M. Extracerebral hematomas in children. In McLaurin RL (ed): Advances in Neurotraumatology, Vol. 1. Wien: Springer 1986, p 173-208.

4. Dhellemm ESP, Lejueune JP, Christaens JL, Combelles G. Traumatic extradural hematomas in infancy and childhood. J Neurosurg 1985, 62:861-864.

5. Mazza C, Pasqualin A, Ferrio HIG, DaPion R, Traumatic extradural hematomas in children: experience with 62 cases. Acta Neurochir (Wien) 1982, 65:67-80.

6. May PL, Miles JB. Acute isodense extradural hematoma. Br J Neurosurg 1989, 3:221-224.

7. Milo R, Razon N Schiffer J. Delayed epidural hematoma: a review. Acta Neurochir (Wien) 1987, 84:13-23.

8. Milza PG, Nardi PV, Gigli R, Motta SA. Epidural hematomas in infancy and childhood: report on 176 cases. J Ped Neurosci 1989, 5:117-122.

9. Molloy CJ, McCaul KA, McLean AJ, North JB, Simpson DA. Extradural haemorrhage in infancy and childhood. Child's Nerv Syst 1990, 6:383-387.

10. Smith HK, Miller JD. The danger of an ultra-early computed tomographic scan in a patient with an evolving acute epidural hematoma. Neurosurgery 1991, 29:258-260. 